\title{
Phase II Trial of Neoadjuvant/adjuvant Imatinib Mesylate for Advanced Primary and Metastatic/recurrent Operable Gastrointestinal Stromal Tumors: Long-term Follow-up Results of Radiation Therapy Oncology Group 0132
}

\author{
Dian Wang, MD, PhD ${ }^{1}$, Qiang Zhang, PhD², Charles D. Blanke, $\mathrm{MD}^{3}$, George D. Demetri, \\ MD $^{4}$, Michael C. Heinrich, MD ${ }^{5}$, James C. Watson, MD ${ }^{6}$, John P. Hoffman, MD ${ }^{6}$, Scott \\ Okuno, MD ${ }^{7}$, John M. Kane, MD $^{8}$, Margaret von Mehren, MD $^{6}$, and Burton L. Eisenberg, MD $^{9}$ \\ ${ }^{1}$ Medical College of Wisconsin, Milwaukee, WI \\ ${ }^{2}$ Radiation Therapy Oncology Group Statistical Center, Philadelphia, PA \\ ${ }^{3}$ University of British Columbia and British Columbia Cancer Agency, Vancouver, BC, Canada \\ ${ }^{4}$ Dana-Farber Cancer Institute and Harvard Medical School, Boston, MA \\ ${ }^{5}$ Portland VA Medical Center and Oregon Health \& Science University, Portland, OR \\ ${ }^{6}$ Fox Chase Cancer Center, Philadelphia, PA \\ ${ }^{7}$ Mayo Clinic, Rochester, MN \\ ${ }^{8}$ Roswell Park Cancer Institute, Buffalo, NY \\ ${ }^{9}$ Dartmouth-Hitchcock Medical Center, Lebanon, NH
}

\begin{abstract}
Background-Imatinib inhibits the KIT and PDGFR tyrosine kinases, resulting in its notable antitumor activity in gastrointestinal stromal tumor (GIST). We previously reported the early results of a multi-institutional prospective trial (RTOG 0132) using neoadjuvant/adjuvant imatinib either in primary resectable GIST or as a planned preoperative cytoreduction agent for metastatic/ recurrent GIST.
\end{abstract}

\begin{abstract}
Methods-Patients with primary GIST ( $25 \mathrm{~cm}$, group A) or resectable metastatic/recurrent GIST ( $\geq 2 \mathrm{~cm}$, group B) received neoadjuvant imatinib $(600 \mathrm{mg} /$ day) for approximately 2 months and maintenance postoperative imatinib for 2 years. We have now updated the clinical outcomes including progression-free survival, disease-specific survival, and overall survival at a median follow-up of 5.1 years, and we correlate these end points with duration of imatinib therapy.

Results-Sixty-three patients were originally entered (53 analyzable: 31 in group A and 22 in group B). Estimated 5-year progression-free survival and overall survival were 57\% in group A, $30 \%$ in group B; and $77 \%$ in group A, $68 \%$ in group B, respectively. Median time to progression has not been reached for group A and was 4.4 years for group B. In group A, in 7 of 11 patients, disease progressed $>2$ years from registration; 6 of 7 patients with progression had stopped imatinib before progression. In group B, disease progressed in 10 of 13 patients $>2$ years from
\end{abstract}

(C) Society of Surgical Oncology 2011

D. Wang, MD, PhD, dwang@mcw.edu.

Presented in part at the 47th annual meeting of American Society of Clinical Oncology, June 3-7, 2011, Chicago, IL. 
registration; 6 of 10 patients with progressing disease had stopped imatinib before progression. There was no significant increase in toxicity compared with our previous shortterm analysis.

Conclusions-This long-term analysis suggests a high percentage of patients experienced disease progression after discontinuation of 2-year maintenance imatinib therapy after surgery. Consideration should be given to studying longer treatment durations in intermediate- to high-risk GIST patients.

Management strategies for gastrointestinal stromal tumor (GIST) have greatly evolved in the past decade. ${ }^{1,2}$,The recognition of activating mutations in the KIT (80-95\%) and/or PDGFR-a (5\%) tyrosine kinases, and the introduction of targeted tyrosine kinase inhibitor (TKI) therapies, have revolutionized the management of these patients. ${ }^{3-5}$ The first TKI approved for the treatment of metastatic or locally advanced GIST was imatinib mesylate (Gleevec; Novartis Pharma AG, Basel, Switzerland), a selective receptor tyrosine kinase inhibitor of KIT and PDGFR-a (as well as BCR-ABL). ${ }^{6}$ On the basis of the accumulated data from clinical studies, imatinib was approved as a first-line therapy for locally unresectable or metastatic GISTs and as an adjuvant without specifying specific duration after resection in patients with intermediate- to high-risk primary GISTs. ${ }^{1,7-15}$ Furthermore, the therapeutic role of neoadjuvant imatinib is unclear beyond its possible facilitation of surgical resection and/or possible reduction of tumor spill or bleeding during surgery, as previously mentioned. ${ }^{1,2}$

Radiation Therapy Oncology Group (RTOG) 0132/American College of Radiology Imaging Network (ACRIN) 6665 was one of the prospective studies to investigate the effectiveness of imatinib mesylate on GIST and to contribute data to the management guidelines. Unlike other studies, this multi-institutional prospective phase II study was designed to assess the clinical outcomes and tolerability of imatinib (600 mg/day) provided for 8-12 weeks either as preoperative therapy before a planned resection of intermediate- to high-risk primary GIST, or as a cytoreductive agent before planned resection of metastatic and/or recurrent GIST. All patients planned to continue postoperative imatinib $(600 \mathrm{mg} /$ day) therapy for an extended postoperative period of 2 years. The short-term analysis of the patients enrolled onto this clinical trial showed encouraging results of outcomes with a low rate of toxicity. ${ }^{14}$

In this report, we have now updated the clinical outcomes including progression-free survival (PFS), diseasespecific survival (DSS), and overall survival (OS), and we correlate these outcomes with the duration of imatinib therapy.

\section{PATIENTS AND METHODS}

The trial design and the rationale for this design were previously reported. ${ }^{14}$ Briefly, the trial eligibility included documented (CD117-expressing KIT positive) GIST patients with either primary disease ( $25 \mathrm{~cm}$ ) or metastatic/recurrent disease (with at least one lesion $\geq 2 \mathrm{~cm}$ ). Patients were treated with imatinib (600 mg/day) for a period of 8-12 weeks before surgery. Imatinib was stopped on the day before surgery and resumed as soon as possible postoperatively (600 mg/day) for 2 years (Fig. 1). Toward the end of accrual completion, the study was amended to allow continuation of postoperative imatinib in patients with metastatic residual disease at the investigator's discretion. Clinical end points included assessments of imatinib-related toxicity and surgical complication assessment, GIST response to preoperative therapy, time to progression, and survival (PFS, DSS, OS).

Time to progression, PFS, DSS, and OS were measured from the date of registration. Time to progression and DSS were estimated by the cumulative incidence method to account for competing risks. ${ }^{16} \mathrm{PFS}$ and OS were defined in the protocol as previously reported and were estimated with the Kaplan-Meier method. ${ }^{14,17}$ Correlation between resection status and 
disease progression was assessed by Fisher's exact test. Response Evaluation Criteria in Solid Tumors (RECIST, v.1.0) was used to measure objective response to preoperative imatinib. ${ }^{18}$ Toxicities were scored using the Common Toxicity Criteria version 2.0; only events scored as definitely, probably, or possibly related (or unknown relationship) were considered related to protocol treatment. Only patients eligible per protocol criteria who initiated protocol treatment were included in analysis.

\section{RESULTS}

RTOG 0132/ACRIN 6665 was open to accrual from February 2002 through June 2006. Patients were entered from 18 RTOG institutions after institutional review board approval. Sixty-three patients were recruited; 53 had analyzable data. Median age was 58 (range 24$84)$ years. There were 29 men $(55 \%)$ and 24 women $(45 \%) .{ }^{14}$ There were 31 patients $(58 \%)$ in group A (primary GIST) and 22 patients (42\%) in group B (metastatic and/or recurrent GIST). Most primary GIST presented in the stomach (52\%), followed by small bowel (20\%); for patients with metastatic/recurrent GIST, the most common location of disease at the time of study entry was noted as "abdominal/peritoneum" (50\%) (Table 1). The median tumor size in group A was 8.7 (range 5-24.5) cm, indicating that the disease of patients in this group was consistent with an intermediate to high risk of disease recurrence. ${ }^{4}$

Imatinib was reported to be a well-tolerated drug in the earlier analysis of this study. ${ }^{14}$ Preoperative toxicity profiles, compliance for use of imatinib, and tumor response to imatinib (RECIST) before surgery have been previously reported. ${ }^{14}$ Similarly, surgical complications remain the same as previously reported. ${ }^{14}$ The long-term complication rates associated with the planned 2-year postresection administration of imatinib therapy were slightly increased when compared with the earlier report, now with more follow-up data (Table 2). At the time of the initial report, grade 3, 4, and 5 toxicities had been reported for $(30.8 \%)$ of $52,11(21.2 \%)$ of 52 , and $1(1.9 \%)$ of patients, respectively. For this long-term update, rates of grade 3,4 , and 5 postoperative toxicities were 18 (34.0\%) of 53, $11(20.8 \%)$ of 53, and $1(1.9 \%)$ of 53, respectively. Two patients experienced new grade 3 toxicities (fatigue; fatigue and ileus). Three patients in group A and 4 patients in group B continued imatinib after 2-year protocol therapy. Seven patients in group A and 6 patients in group B reinitiated imatinib after disease progression. Fifteen patients stopped adjuvant therapy early for reasons other than disease progression. In group A, this comprised 9 patients total: 4 due to toxicity, 3 patient refusal, 1 death, and 1 unknown. In group B, this comprised 6 patients total: 3 due to patient refusal, 1 toxicity, 1 alternative treatment, and 1 other disease.

In group A (primary GIST), median follow-up for surviving patients was 4.9 (range 2.7-7.0) years. Eleven patients (35.5\%) experienced disease progression or recurrence, and 10 patients died. The 2- and 5-year estimates of risk of disease progression are 12.9\% [95\% confidence interval (CI) 0.9-24.9] and 36.9\% (95\% CI 19.0-54.8), respectively. Median time to progression has not been yet reached. The 2- and 5-year PFS rate estimates are 83.9\% (95\% CI 70.9-96.8) and 56.7\% (95\% CI 38.7-74.6) (95\% CI; Fig. 2). The 2- and 5year OS rate estimates are $93.5 \%$ (95\% CI 84.8-100) and 76.9\% (95\% CI 61.9-91.9) (95\% CI; Fig. 3). The 2- and 5-year DSS rate estimates are 93.5\% (95\% CI 84.8-100) and 76.9\% (95\% CI 61.7-92.2).

In group B (metastatic/recurrent GIST), median followup for surviving patients was 5.5 (range 2.2-8.0) years. Thirteen patients (59.1\%) had evidence of objective disease progression, and 7 patients died. The 2- and 5-year estimates for risk of disease progression are $13.6 \%$ (95\% CI 0-28.4) and 61.2\% (95\% CI 37.7-84.6). Median time to progression is 4.4 years. The 2- and 5-year PFS rate estimates are $77.3 \%$ (95\% CI 59.8-94.8) and 29.8\% (95\% CI 8.8-50.7) (Fig. 2). The 2- and 5-year OS estimates are 90.9\% (95\% CI 78.9-100) 
and 68.2\% (95\% CI 46.9-89.5) (Fig. 3). The 2- and 5-year DSS estimates are $100 \%$ and $77.3 \%$ (95\% CI 57.0-97.5).

Correlation between surgical resection (R) status and tumor progression has been assessed (Table 3). For group A, there is some indication, of an association between $\mathrm{R}$ status and tumor progression, but the difference is not statistically significant [no surgery, R1-2: 6 $(60.0 \%)$ of 10 , vs. R0: 5 (23.8\%) of $21 ; P=0.11$ ]. However, for group B, there does not appear to be a correlation between $\mathrm{R}$ status and tumor progression (Table 3 ). These data, relative to $\mathrm{R}$ status, must be interpreted in the context of a small patient sample in each subgroup. Estimated 5-year OS of all patients in both group A and group B is $80.2 \%$ for patients with R0 resection and $73.4 \%$ for patients with $\mathrm{R} 1-2$ resection (hazard ratio 1.33; 95\% CI $0.40-4.47 ; P=0.64)$.

A correlation between duration of imatinib therapy and tumor progression was also assessed. In group $\mathrm{A}$, disease of 7 of 11 patients progressed more than 2 years from study registration; 6 of 7 patients with progressive disease had stopped imatinib therapy before progression. In group B, 10 of 13 patients experienced progression more than 2 years from registration; 6 of 10 patients with progression had stopped imatinib therapy before progression.

\section{DISCUSSION}

Imatinib has been accepted as the standard first line systemic therapy for patients with metastatic and/or unresectable, locally advanced GIST based on the accumulated data derived from large prospective studies. ${ }^{1,2,19}$ Imatinib therapy (400 mg/day) has been routinely recommended on the basis of these data, with the potential to increase the dose to $800 \mathrm{mg} /$ day if disease progressed or if a mutation in exon 9 of KIT is identified. The RTOG 0132/ACRIN 6665 trial was conceptualized in 2001 and began patient accrual in February 2002 when optimal dose and duration of imatinib therapy for GIST patients was not yet standardized. The study was designed for postoperative imatinib therapy at $600 \mathrm{mg} /$ day for 2 years, although it has since become clear from many lines of evidence, such as the French Sarcoma Group BFR14 trial, that stopping imatinib in patients with metastatic disease whose disease experienced response after multimodal therapy is associated with rapid disease recurrence and progression. ${ }^{20}$ The data from this trial further support this finding: 10 of 13 group B patients experienced disease progression more than 2 years from registration, and $6(60 \%)$ of 10 of the patients with progression had stopped imatinib therapy before documented progression. This supports current recommendations that all patients with metastatic and/or unresectable GIST should continue imatinib therapy indefinitely until evidence of disease progression. ${ }^{1,2,19}$

It is important to note that the median time to progression is 4.4 years in this group, which is considerably longer than the 24 months reported from other trials of metastatic GIST. This lengthy time to progression cannot be explained solely by the success of surgical resection of metastatic/recurrent GIST in this cohort because the complete resection (R0) or maximal debulking (R1) of metastatic/recurrent GIST could not be definitively demonstrated to have a positive impact on tumor progression with comparison to those with gross tumor left behind (R2) or to those with no surgery (Table 3 ). This may partially be explained by the small number of patients in this group $(n=22)$ as well as by the fact that patients in this group were specifically chosen to be potentially amenable to surgical resection, thus likely demonstrating less tumor volume upon study entry, and certainly reflecting a more highly selected group than the larger phase 2 or 3 studies that included patients with widespread, bulky metastatic disease who were specifically judged to have surgically unresectable disease. Indeed, data from U.S. Finish Study B2222 in advanced GIST evaluated tumor volume to outcome, with lower tumor burden correlating with improved disease control. ${ }^{9}$ 
Surgery has long been the cornerstone in the management of localized GIST, with reported 5 -year recurrence-free and disease-free survival rates of $49 \%$ and $65 \%$ after complete resection. ${ }^{21,22}$ Recently, imatinib has been approved by regulatory authorities as adjuvant therapy for adult patients with primary GIST who are at high risk of relapse after resection. ${ }^{1,2,19}$ This approval is based on the results from the phase 3 American College of Surgery Oncology Group (ACOSOG) Z9001 trial of 778 patients with KIT-expressing GIST ( $33 \mathrm{~cm}$ ) who underwent complete resection followed by 1 year of imatinib ( $400 \mathrm{mg} /$ day) or placebo. It is relevant to note that the approval language of the U.S. Food and Drug Administration did not specify duration for imatinib dosing in the adjuvant setting, and thus the approval did not match the duration for which imatinib was administered on this pivotal trial. ${ }^{7}$ Adjuvant imatinib provided a significant improvement in recurrence-free survival but not OS in short-term follow-up of the Z9001 trial. From the long-term update of RTOG 01 of 31 patients with GIST of at least intermediate risk (based purely on tumor size-median size $8.7 \mathrm{~cm}$, range $5-24.5 \mathrm{~cm}$ ) who completed resection followed by adjuvant imatinib therapy for 2 years (group A), we noted that complete resection of gross and microscopic disease (R0) had an impact on time to progression. This confirms the expected importance of the R0 resection in the management of localized GIST. ${ }^{23-25}$ In addition, a significant drop is noted in both PFS and OS after 2 years when adjuvant imatinib therapy was discontinued (see Fig. 2 for PFS and Fig. 3 for OS). For example, PFS drops from of $83.9 \%$ at 2 years to $67.4 \%$ at 3 years, then to $60.7 \%$ at 4 years, and finally to $56.7 \%$ at 5 years. Furthermore, 7 of 11 patients exhibited progression of GIST more than 2 years from registration; 6 of 7 patients with disease progression had stopped imatinib therapy before progression. These results suggest that a longer duration of adjuvant imatinib therapy is required to delay the development of recurrence/metastasis and to prolong PFS in patients with intermediate- to high-risk GIST. We hope that long-term follow-up of the ACOSOG Z9001 trial will confirm our observation. A recent finding was reported by the SSG XVII/AIO in the 2011 ASCO proceedings. This 400 patient prospective randomized trial of adjuvant imatinib administered post-operatively for 12 months versus 36 months in high-risk primary GIST patients indicated both a disease-free survival and OS benefit for the 36 month duration group. ${ }^{26}$

Results from several small retrospective studies have suggested that some patients with localized metastatic/recurrent GIST may benefit from surgery after response or stabilization to imatinib treatment. ${ }^{1,2,19,27-30}$ In these retrospective studies, preoperative imatinib therapy was provided for 3-12 months. It is now commonly recommended that resection should be performed once the maximum benefit from imatinib has been achieved but before tumor progression occurs. However, the clinical role of imatinib therapy followed by surgical resection in responsive metastatic GIST remains intriguing but unproven. To our knowledge, RTOG 0132 is the only reported multi-institutional prospective study to evaluate the activity and tolerability of imatinib (600 mg/day for 8-12 weeks) as a preoperative cytoreductive agent before a planned resection of metastatic/recurrent GIST. Results from this long-term analysis show it is safe to have a surgical resection after preoperative imatinib therapy for 812 weeks, but suggests that the complete resection (R0) or maximal debulking (R1) in this small group of patients had no substantial impact on tumor progression compared to patients with no surgery or gross residual tumor left behind (R2) (Table 3). It therefore still remains an open question as to benefit of an extensive abdominal surgery in imatinib-responsive disease or in stable patients with metastatic GIST in the absence of tumor-related symptoms. A prospective clinical trial addressing this issue was launched in Europe, but unfortunately it was closed as a result of poor accrual. Therefore, the absolute role of surgical resection in metastatic/recurrent GIST in the imatinib era is not well defined. The potential advantage of tumor removal in this setting is to obviate drug resistance or to prevent development of complications from a large metastatic site. Individual early on multidisciplinary management for these patients is critical to decisions regarding the combination of surgery 
and imatinib. Defined variables, such as tumor volume, and as yet undefined variables, such as tumor genomic profiling, may prove to be important in these decisions.

Similarly, the role of neoadjuvant imatinib for patients with locally advanced, though resectable, GIST remains a matter of judgement for a multidisciplinary team to use in shared decision making with a patient. The short-term analysis from RTOG 0132/ACRIN 6665 demonstrated the safety and feasibility of neoadjuvant imatinib therapy $(600 \mathrm{mg} /$ day for $8-$ 12 weeks) before surgery. ${ }^{14}$ Furthermore, we noted that patients who had no surgery or had R1-2 resection trended toward having a higher chance of developing tumor progression compared with patients who had an R0 resection $(60 \%$ vs. $23.8 \%, P=0.11)$. A publication from the French Sarcoma Group BFR14 study identified 25 patients with locally advanced nonmetastatic/recurrent GIST who were treated with neoadjuvant imatinib. ${ }^{31}$ The resection group (9 patients) had marked advantage in terms of PFS and OS when compared with the 16 patients who did not undergo resection of their primary GIST. Although this might simply be due to other clinical and biological differences between these patient groups, it is also consistent with a possible impact of neoadjuvant and adjuvant imatinib as well as complete surgical resection of the primary GIST. Neoadjuvant imatinib may be important in clinical circumstances where tumor shrinkage will facilitate complete resection or decrease the morbidity of the surgical resection. It might also be clinically relevant to know whether the GIST responds to imatinib therapy before extensive abdominal surgery. However, because of the small sample size and nonrandomized study design, the results of RTOG 0132 or BFR14 fail to provide definitive evidence that neoadjuvant sequencing adds more benefit than adjuvant imatinib alone for patients with resectable primary GIST with intermediate- to high-risk features.

In conclusion, the long-term analysis of the patients enrolled onto RTOG 0132/ACRIN 6665 showed no significant increase in treatment complications after preoperative imatinib therapy (600 mg/day for 2-3 months) in patients with resectable locally advanced GIST (group A) or patients with operable metastatic/recurrent GIST (group B) followed by maintenance therapy $(600 \mathrm{mg} / \mathrm{day})$ for 2 years. Complete resection of gross and microscopic disease (R0) appears to have an impact on tumor progression in patients with resectable locally advanced GIST, but this was not demonstrated in patients with metastatic/recurrent GIST. However, the analysis was certainly affected by the small number of patients in the metastatic/recurrent GIST group, particularly those with R2 or no surgery, and our analysis cannot provide any important conclusion regarding the benefit, or lack thereof, with planned surgical resection. In addition, the disease of a high percentage of patients progressed after discontinuation of 2-year maintenance imatinib therapy after surgical resection, supporting the need to study longer treatment durations of imatinib in patients with primary GIST after resection yet who are at a high risk of disease recurrence as per modern risk profiling of the disease.

\section{Acknowledgments}

Supported in part by grants RTOG UCA21661 and CCOP U10 CA37422 from the National Cancer Institute (NCI). The contents of this article are the sole responsibility of the authors and do not necessarily represent the official views of the NCI. The authors received consulting fees, honoraria, and research support as follows: Consulting: Charles D. Blanke (Novartis), George D. Demetri (Novartis), Margaret von Mehren (Novartis), Burt Eisenberg (Novartis), Michael Heinrich (Novartis, MolecularMD). Honoraria: Charles D. Blanke (Novartis), George D. Demetri (Novartis), Michael Heinrich (Novartis), Margaret. von Mehren (Novartis), Burt Eisenberg (Novartis). Research support: George D. Demetri (Novartis), Margaret. von Mehren (Novartis). Michael Heinrich (Novartis, Ariad, Lilly, AROG Pharmaceuticals), Scott Okuno (Novartis). Stock ownership: Michael Heinrich (MolecularMD). 


\section{REFERENCES}

1. Demetri GD, von Mehren M, Antonescu CR, et al. NCCN Task Force report: update on the management of patients with gastrointestinal stromal tumors. J Natl Compr Canc Network. 2010; 8:S1-S41.

2. Casali PG, Jost L, Reichardt P, et al. Gastrointestinal stromal tumours: ESMO clinical recommendations for diagnosis, treatment and follow-up. Ann Oncol. 2009; 20(Suppl 4):64-67. [PubMed: 19454466]

3. Corless CL, Heinrich MC. Molecular pathobiology of gastrointestinal stromal sarcomas. Annu Rev Pathol. 2008; 3:557-586. [PubMed: 18039140]

4. Fletcher CD, Berman JJ, Corless C, et al. Diagnosis of gastrointestinal stromal tumors: a consensus approach. Hum Pathol. 2002; 33:459-465. [PubMed: 12094370]

5. Heinrich MC, Corless CL, Duensing A, et al. PDGFRA activating mutations in gastrointestinal stromal tumors. Science. 2003; 299(5607):708-710. [PubMed: 12522257]

6. Demetri GD, von Mehren M, Blanke CD, et al. Efficacy and safety of imatinib mesylate in advanced gastrointestinal stromal tumors. N Engl J Med. 2002; 347:472-480. [PubMed: 12181401]

7. Dematteo RP, Ballman KV, Antonescu CR, et al. Adjuvant imatinib mesylate after resection of localised, primary gastrointestinal stromal tumour: a randomised, double-blind, placebocontrolled trial. Lancet. 2009; 373(9669):1097-104. [PubMed: 19303137]

8. Blanke CD, Rankin C, Demetri GD, et al. Phase III randomized, intergroup trial assessing imatinib mesylate at two dose levels in patients with unresectable or metastatic gastrointestinal stromal tumors expressing the kit receptor tyrosine kinase: S0033. J Clin Oncol. 2008; 26:626-632. [PubMed: 18235122]

9. Blanke CD, Demetri GD, von Mehren M, et al. Long-term results from a randomized phase II trial of standard- versus higher-dose imatinib mesylate for patients with unresectable or metastatic gastrointestinal stromal tumors expressing KIT. J Clin Oncol. 2008; 26:620-625. [PubMed: 18235121]

10. Verweij J, Casali PG, Zalcberg J, et al. Progression-free survival in gastrointestinal stromal tumours with high-dose imatinib: randomised trial. Lancet. 2004; 364(9440):1127-1134. [PubMed: 15451219]

11. Zalcberg JR, Verweij J, Casali PG, et al. Outcome of patients with advanced gastro-intestinal stromal tumours crossing over to a daily imatinib dose of $800 \mathrm{mg}$ after progression on $400 \mathrm{mg}$. Eur J Cancer. 2005; 41:1751-1757. [PubMed: 16098458]

12. Debiec-Rychter M, Sciot R, Le Cesne A, et al. KIT mutations and dose selection for imatinib in patients with advanced gastrointestinal stromal tumours. Eur J Cancer. 2006; 42:1093-1103. [PubMed: 16624552]

13. Le Cesne A, Van Glabbeke M, Verweij J, et al. Absence of progression as assessed by response evaluation criteria in solid tumors predicts survival in advanced GI stromal tumors treated with imatinib mesylate: the intergroup EORTC-ISG-AGITG phase III trial. J Clin Oncol. 2009; 27:3969-3974. [PubMed: 19620483]

14. Eisenberg BL, Harris J, Blanke CD, et al. Phase II trial of neo-adjuvant/adjuvant imatinib mesylate (IM) for advanced primary and metastatic/recurrent operable gastrointestinal stromal tumor (GIST): early results of RTOG 0132/ACRIN 6665. J Surg Oncol. 2009; 99:42-47. [PubMed: 18942073]

15. McAuliffe JC, Hunt KK, Lazar AJ, et al. A randomized, phase II study of preoperative plus postoperative imatinib in GIST: evidence of rapid radiographic response and temporal induction of tumor cell apoptosis. Ann Surg Oncol. 2009; 16:910-919. [PubMed: 18953611]

16. Kalbfleisch, JD.; Prentice, RL. The statistical analysis of failure time data. New York: Wiley; 1980.

17. Kaplan EL, Meier P. Nonparametric estimation from incomplete observations. J Am Stat Assoc. $1958 ; 53: 457$.

18. Therasse P, Arbuck S, Eisenhauer E, et al. New guidelines to evaluate the response to treatment in solid tumors. J Natl Cancer Inst. 2000; 92:205-216. [PubMed: 10655437] 
19. Blackstein ME, Blay JY, Corless C, et al. Gastrointestinal stromal tumours: consensus statement on diagnosis and treatment. Can J Gastroenterol. 2006; 20:157-163. [PubMed: 16550259]

20. Le Cesne A, Ray-Coquard I, Bui BN, et al. Discontinuation of imatinib in patients with advanced gastrointestinal stromal tumours after 3 years of treatment: an open-label multicentre randomised phase 3 trial. Lancet Oncol. 2010; 11:942-949. [PubMed: 20864406]

21. Hassan I, You YN, Shyyan R, et al. Surgically managed gastrointestinal stromal tumors: a comparative and prognostic analysis. Ann Surg Oncol. 2008; 15:52-59. [PubMed: 18000711]

22. Singer S, Rubin BP, Lux ML, et al. Prognostic value of KIT mutation type, mitotic activity, and histologic subtype in gastrointestinal stromal tumors. J Clin Oncol. 2002; 20:3898-3905. [PubMed: 12228211]

23. Miettinen M, Lasota J. Gastrointestinal stromal tumors: review on morphology, molecular pathology, prognosis, and differential diagnosis. Arch Pathol Lab Med. 2006; 130:1466-1478. [PubMed: 17090188]

24. Joensuu H. Risk stratification of patients diagnosed with gastrointestinal stromal tumor. Hum Pathol. 2008; 39:1411-1419. [PubMed: 18774375]

25. Dematteo RP, Gold JS, Saran L, et al. Tumor mitotic rate, size, and location independently predict recurrence after resection of primary gastrointestinal stromal tumor (GIST). Cancer. 2008; 112:608-615. [PubMed: 18076015]

26. Joensuu H. Twelve versus 36 months of adjuvant imatinib (IM) as treatment of operable GIST with a high risk of recurrence: final results of randomized trial (SSGXVIII/AIO). J Clin Oncol. 2011; (18 Suppl):29.

27. Andtbacka RH, Ng CS, Scaife CL, et al. Surgical resection of gastrointestinal stromal tumors after treatment with imatinib. Ann Surg Oncol. 2007; 14:14-24. [PubMed: 17072676]

28. Bonvalot S, Eldweny H, Péchoux CL, et al. Impact of surgery on advanced gastrointestinal stromal tumors (GIST) in the imatinib era. Ann Surg Oncol. 2006; 13:1596-1603. [PubMed: 16957966]

29. Rutkowski P, Nowecki Z, Nyckowski P, et al. Surgical treatment of patients with initially inoperable and/or metastatic gastrointestinal stromal tumors (GIST) during therapy with imatinib mesylate. J Surg Oncol. 2006; 93:304-311. [PubMed: 16496358]

30. Raut CP, Posner M, Desai J, et al. Surgical management of advanced gastrointestinal stromal tumors after treatment with targeted systemic therapy using kinase inhibitors. J Clin Oncol. 2006; 24:2325-2331. [PubMed: 16710031]

31. Blesius A, Cassier PA, Bertucci F, et al. Neoadjuvant IM in patients with locally advanced GIST in the prospective BFR14 trial. BMC Cancer. 2011; 11:72. [PubMed: 21324142] 


\section{RTOG/ACRIN 6665 Schema}

\section{KIT positive GIST}

Group A: primary locally advanced $(\geq 5 \mathrm{~cm})$

Group B: operable recurrent/metastatic $(\geq 2 \mathrm{~cm})$

\section{Baseline PET and CT}

\section{Imatinib $600 \mathrm{mg} /$ day for 8-12 weeks}

\section{Repeat PET and CT}

\section{Resection}

Continued Imatinib

$600 \mathrm{mg} /$ day for

24 hours

FIG. 1.

Schematic representation of the trial. $S D$ stable disease, $P R$ partial response, $P D$ progressive disease 


\section{Progression-free survival (\%)}

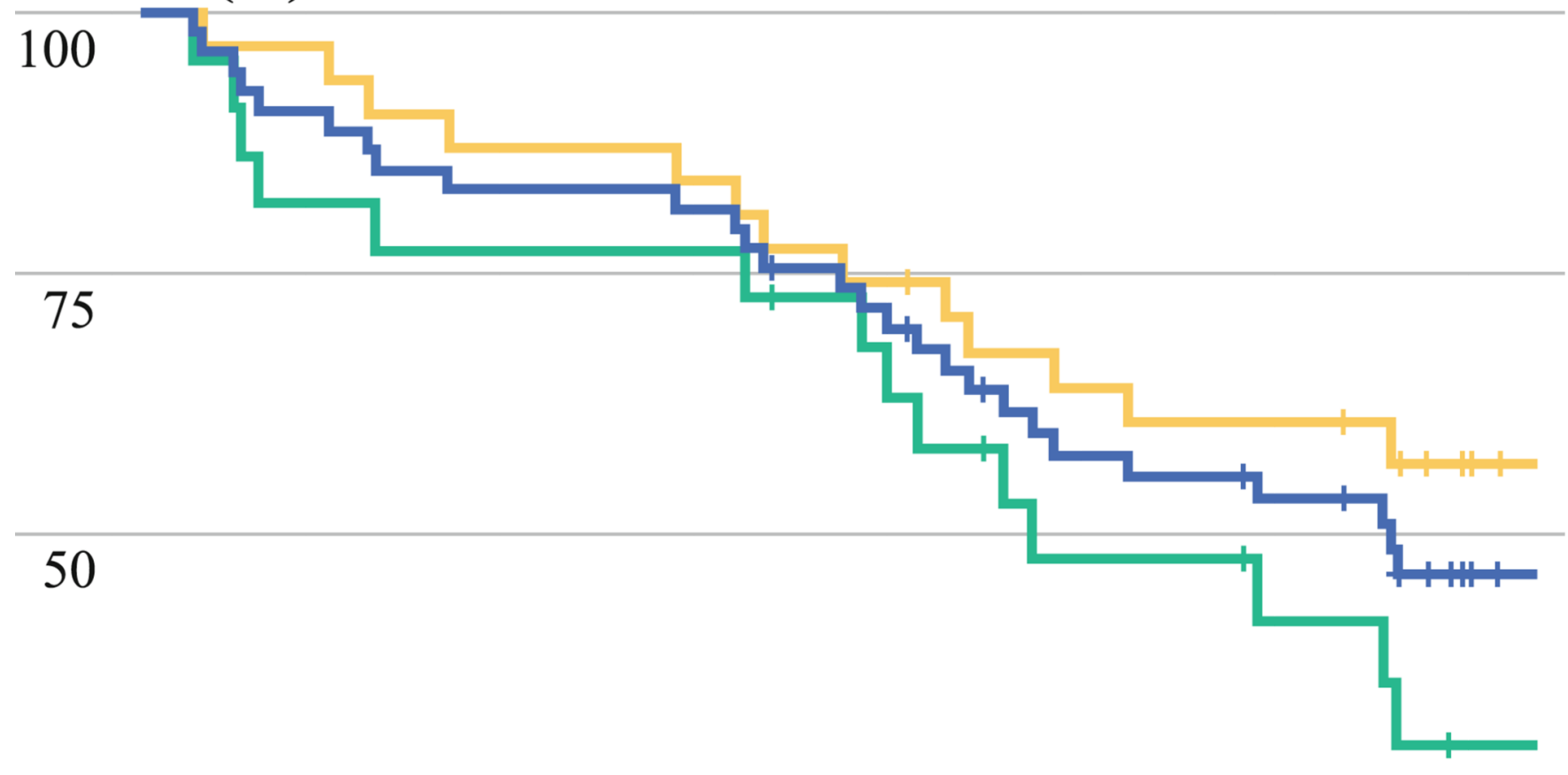

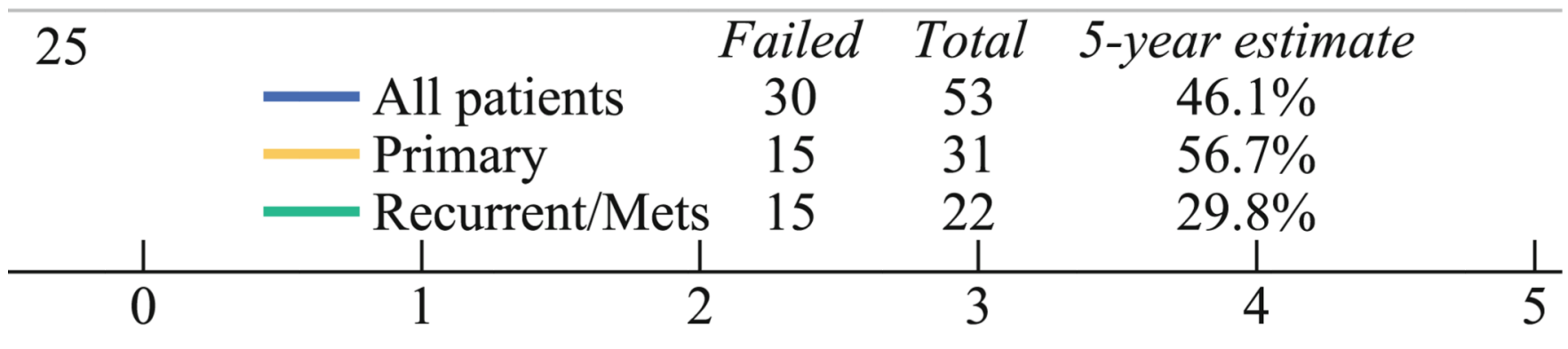

Patients at risk

\section{Years after registration}

$\begin{array}{rr}53 & 45 \\ -31 & 28 \\ -22 & 17\end{array}$

FIG. 2.

Progression-free survival

31
20
11

25

18

7 


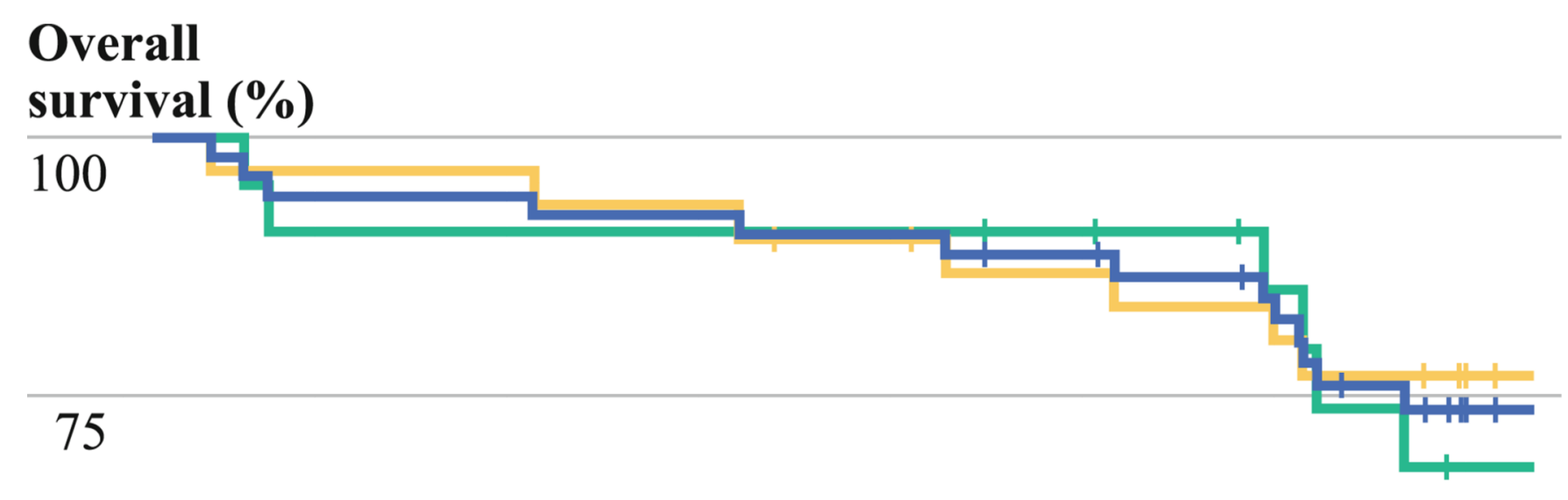

\section{0}

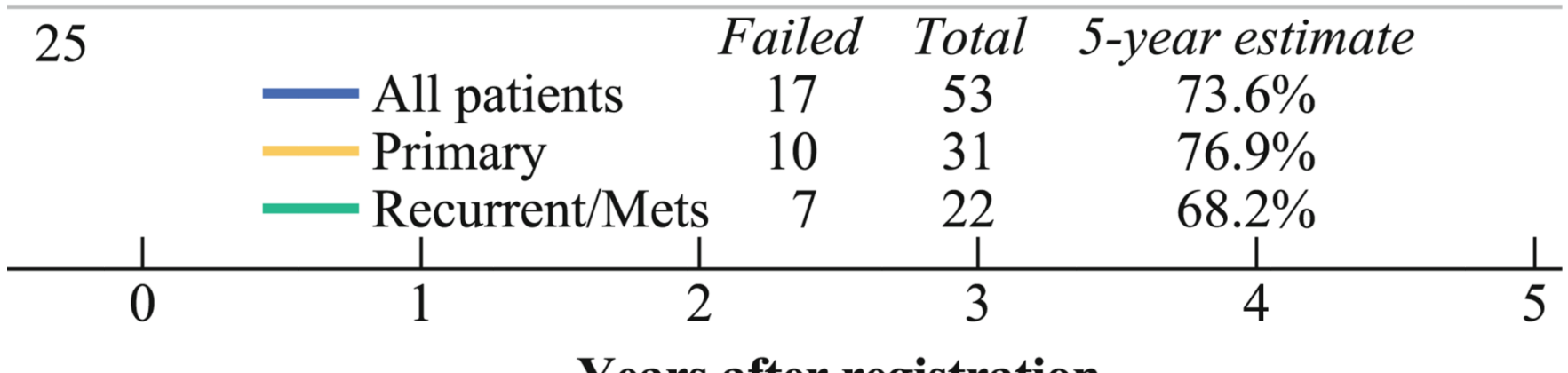

Patients at risk

\section{Years after registration}

$\begin{array}{rrrrrr}53 & 50 & 49 & 44 & 41 & 24 \\ -31 & 30 & 29 & 26 & 25 & 13 \\ -22 & 20 & 20 & 18 & 16 & 11\end{array}$

FIG. 3.

Overall survival 
TABLE 1

Patient and tumor characteristics

\begin{tabular}{|c|c|c|}
\hline Characteristic & $\begin{array}{l}\text { Patients with } \\
\text { primary disease } \\
(n=31)\end{array}$ & $\begin{array}{l}\text { Patients with } \\
\text { recurrent or } \\
\text { metastatic disease } \\
(n=22)\end{array}$ \\
\hline \multicolumn{3}{|l|}{ Age $(y)$} \\
\hline Median & 63 & 51.5 \\
\hline Min-max & $42-84$ & $24-77$ \\
\hline Quartiles 1-3 & $54-71$ & $45-59$ \\
\hline \multicolumn{3}{|l|}{ Gender } \\
\hline Male & $16(51.6 \%)$ & $13(59.1 \%)$ \\
\hline Female & $15(48.4 \%)$ & $9(40.9 \%)$ \\
\hline \multicolumn{3}{|l|}{ Zubrod performance status } \\
\hline 0 & $10(32.3 \%)$ & $19(86.4 \%)$ \\
\hline 1 & $18(58.1 \%)$ & $3(13.6 \%)$ \\
\hline 2 & $3(9.7 \%)$ & $0(0.0 \%)$ \\
\hline \multicolumn{3}{|l|}{ Disease size (longest diameter, $\mathrm{cm}$ ) } \\
\hline Median & 8.7 & 5.5 \\
\hline Min-max & $5.0-24.5$ & $2.0-15.5$ \\
\hline Quartiles 1-3 & $6.2-15.2$ & $3.8-8.7$ \\
\hline \multicolumn{3}{|l|}{ Disease location } \\
\hline Abdomen & $1(3.2 \%)$ & $1(4.5 \%)$ \\
\hline Duodenum & $1(3.2 \%)$ & $0(0.0 \%)$ \\
\hline Intra-abdominal periumbilical & $1(3.2 \%)$ & $0(0.0 \%)$ \\
\hline Large intestine & $1(3.2 \%)$ & $0(0.0 \%)$ \\
\hline Liver & $0(0.0 \%)$ & $6(27.3 \%)$ \\
\hline Liver and perirectum & $0(0.0 \%)$ & $1(4.5 \%)$ \\
\hline Pancreas & $1(3.2 \%)$ & $1(4.5 \%)$ \\
\hline Pelvis & $3(9.7 \%)$ & $2(9.1 \%)$ \\
\hline Perirectum & $2(6.5 \%)$ & $0(0.0 \%)$ \\
\hline Peritoneum & $0(0.0 \%)$ & $1(4.5 \%)$ \\
\hline Rectum & $1(3.2 \%)$ & $0(0.0 \%)$ \\
\hline Small intestine & $4(12.9 \%)$ & $1(4.5 \%)$ \\
\hline Stomach & $16(51.6 \%)$ & $9(40.9 \%)$ \\
\hline
\end{tabular}




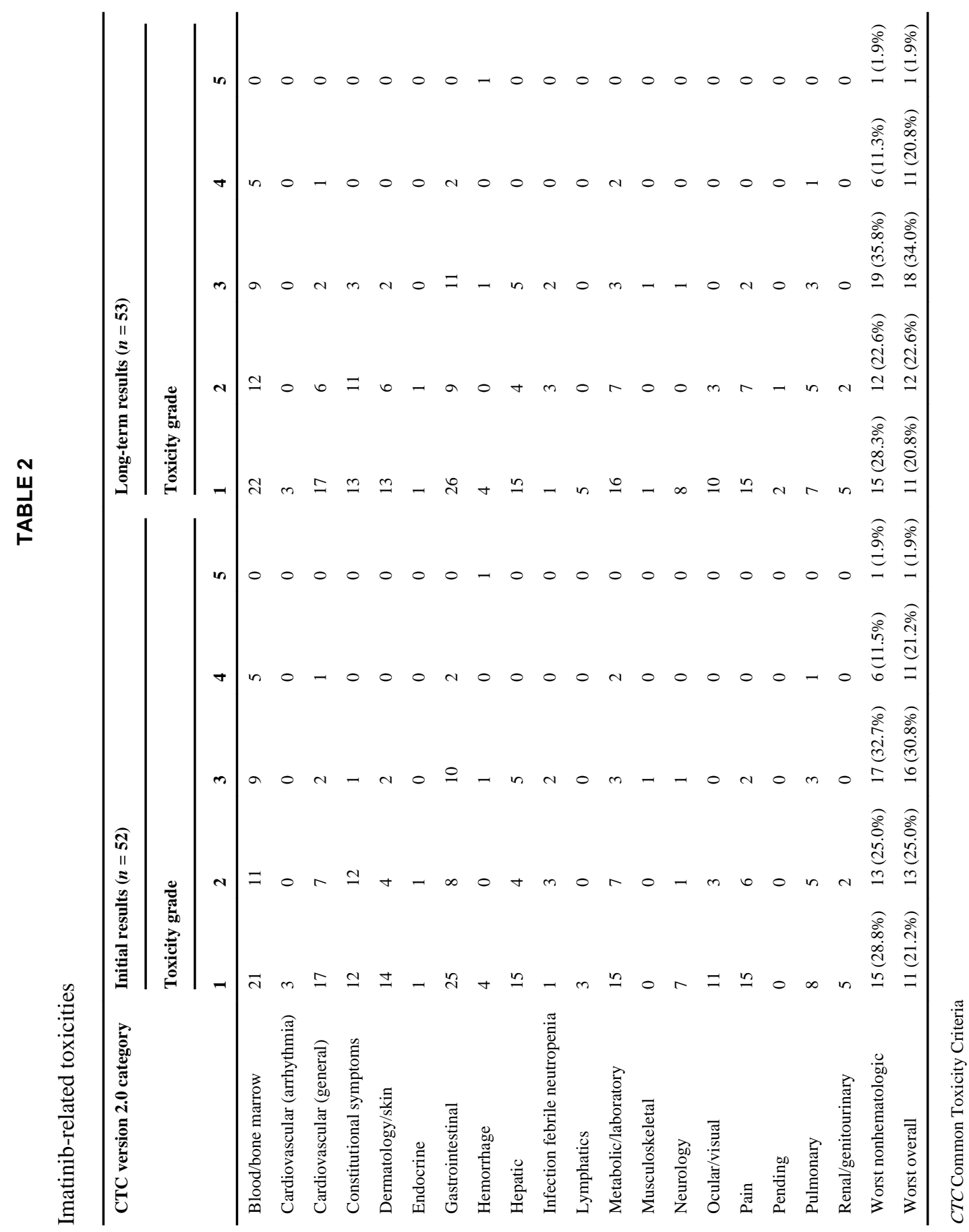


TABLE 3

Correlation between status of surgical resection and tumor progression

\begin{tabular}{ll}
\hline Status & Progression \\
\hline Group A (advanced primary GISTs, resectable) $(n=31)$ & \\
R0 & $5 / 21(23.8 \%)$ \\
R1 & $2 / 4(50.0 \%)$ \\
R2 (gross disease left behind) & $2 / 2(100 \%)$ \\
No surgery & $2 / 4(50.0 \%)$ \\
Group B (metastatic/recurrent disease, potentially operable) $(n=22)$ & \\
R0 & $7 / 11(63.6 \%)$ \\
R1 & $1 / 2(50.0 \%)$ \\
R2 (gross disease left behind) & $3 / 5(60.0 \%)$ \\
No surgery & $1 / 3(33.3 \%)$ \\
Unknown & $1 / 1(100 \%)$ \\
\hline
\end{tabular}

GIST gastrointestinal stromal tumor 